\title{
El perfil directivo femenino y su relación con la orientación al mercado y el desempeño organizacional
}

\author{
Ingrid E. Orlandini \\ Universidad Mayor Real y Pontificia de San Francisco Xavier de Chuquisaca, Facultad de Ciencias Económicas y \\ Empresariales, Calle Padilla s/n Urbanización los Álamos, Sucre, Bolivia. (correo-e: iorlandinig@gmail.com)
}

Recibido Dic. 26, 2019; Aceptado Feb. 28, 2020; Versión final Abr. 1, 2020, Publicado Jun. 2020

\begin{abstract}
Resumen
El objetivo de este estudio es medir la relación entre el perfil femenino, constructo formado por el capital humano y el capital social, la orientación al mercado (cliente, competencia, y coordinación interfuncional) y el desempeño organizacional medido en tres dimensiones: económica, social y ambiental. Se investigó una muestra de 190 mujeres gerentes de empresas hoteleras en dos ciudades patrimoniales de Bolivia (Sucre y Potosí). El estudio es de corte transversal y la escala usada fue Likert. Para calcular las relaciones se aplicó la modelación de ecuaciones estructurales con mínimos cuadrados parciales. Los resultados muestran una relación significativa entre el perfil directivo femenino con la orientación al mercado y con el desempeño organizacional. Se concluye que los gobiernos locales deben impulsar el sector hotelero pues constituye un potencial económico de las ciudades incluidas en este estudio, generandor entornos amigables que coadyuven a la mejora del desempeño de las mujeres directivas.
\end{abstract}

\section{The female managerial profile and its relationship with market orientation and organizational performance}

\begin{abstract}
The objective of this study is to measure the relationship between the female profile, a construct formed by human capital and social capital, market orientation (customer orientation, competition orientation, and cross-functional coordination), and organizational performance measured (economic, social, and environmental). A sample of 190 female hotel managers from two Bolivian heritage cities (Sucre and Potosí) was investigated. This study is cross-sectional in nature and the scale used was Likert. Structural equation modeling with partial least squares was applied to determine the relationship between variables. The results show a significant relationship between the female managerial profile with market orientation and organizational performance. It is concluded that local governments must promote the hotel sector due to its economic potential in the cities included in this study. In addition, governments must generate friendly environments that contribute to improving the performance of female managers.
\end{abstract}




\section{INTRODUCCIÓN}

La relación entre el perfil directivo femenino (PF), la orientación al mercado (OM) y el desempeño organizacional (DO) ha sido estudiada ya sea destacando la importancia del PF y su repercusión en la OM (Behrens y Patzelt, 2016; Berkhout et. al., 2016), analizando la relación entre OM y DO (Alsos y Ljunggren, 2017; Baù et. al., 2017) o midiendo la influencia de la OM con el DO (McKelvie et. al., 2018; Lomberg et. al., 2017) sin embargo, son pocos los análisis que incluyen un enfoque de género y menos el desarrollado de un modelo cognitivo que explique la relación entre las tres variables; por ello se plantea la siguiente pregunta: ¿Cómo influye el perfil femenino en la orientación al mercado y en el desempeño organizacional de las empresas del sector hotelero de las ciudades patrimoniales del sur de Bolivia?.

En Bolivia el 37\% que corresponde a 726.647 mujeres no indígenas entre 18 y 64 años son dueñas de negocios (Querejazu et. al., 2014), el turismo en Bolivia constituye la principal fuente de generación de divisas además de constituirse en la primera actividad económica de ciudades con menos de 300.000 habitantes que se encuentran aisladas del centro político y económico del país como son las ciudades de Sucre y Potosí, el sector hotelero en estas ciudades tiene una incidencia directa y significativa con el PIB, este dato es coincidente con estudios sobre turismo que indican que esta actividad constituye un importante aporte para el desarrollo económico local (Santamaria y Filis, 2019); el 85\% de los hoteles están bajo la administración de mujeres como gerentes-propietarias. De ahí que en este artículo se plantea el objetivo de medir la relación del perfil directivo femenino, la orientación al mercado y el desempeño organizacional en el contexto de la industria hotelera de ciudades patrimoniales en el sur de Bolivia.

El perfil directivo femenino, para fines de esta investigación, estará entendido como un constructo formado por el capital humano y el capital social. La teoría del capital humano analiza a la educación como variable determinante del éxito en el mercado laboral, otros autores señalan que se puede definir como el conocimiento que posee, desarrolla y acumula cada persona en su trayectoria de formación académica y su trayectoria laboral, incluyendo los estilos de aprendizaje que asume (Lu y Herremans, 2019; Huggins et. al., 2017; Millán et. al, 2014). El nivel educativo y la formación continua son dos aspectos que determinan un buen nivel de capital humano; Millán et. al., (2014) establece que el capital humano de un individuo está formado por variables como: la educación formal, el entrenamiento, la experiencia específica y la experiencia general; por ello el capital humano está íntimamente ligado a los estilos de aprendizaje que se consideran las estrategias que emplean las personas para asimilar, incorporar y procesar la nueva información (Gómez et. al., 2015).

Por otro lado el concepto de capital social aparece en la década del 90 con los estudios de Bourdieu (2006), para este autor el capital social es como una red de relaciones objetivas, de dominación o subordinación, de complementariedad o antagonismo. Es decir, constituye la red de relaciones que se convierte en un factor crítico en el desarrollo de cualquier negocio (De la Mora et. al., 2020) y sobre todo de los negocios de las mujeres empresarias (Ventura y Quero, 2012). Según Aldana-Bernal y Bernal-Torres (2019) el capital social es definido de acuerdo a tres dimensiones: la estructural que mide la configuración de la red empresarial para generar oportunidades de transacción; el capital cognitivo que representa aspectos de identidad compartida como metas, cultura y lenguaje compartido entre las partes y el capital relacional referido a elementos que permiten establecer y mantener los vínculos entre los miembros de la red asociados con el comportamiento tal como el respeto y la amistad. Las redes individuales ligadas a lazos familiares, principalmente, que son útiles para aminorar la carga familiar y las redes institucionales que facilitan la puesta en marcha y el desarrollo de los negocios constituyen el capital social de las mujeres (Naldi et. al., 2019; Ventura y Quero, 2012; Liñán y Santos, 2006).

La orientación al mercado en cuanto a definición y enfoque aparece con los estudios de Narver y Slater (1990) que tienen una perspectiva cutural, indican que la rentabilidad de la empresa puede derivarse de su grado de orientación hacia el mercado; está integrada por tres componentes principales: orientación al cliente, orientación a la competencia y coordinación interfuncional y plantean la escala MKTOR para medir esta variable. Por su parte los estudios de Kohli y Jaworski (1990) se basan en un enfoque de comportamiento, identifican tres componentes básicos de la orientación al mercado: 1 la generación de información sobre el mercado (consumidores, competidores y entorno) a través de sistemas formales e informales, 2 la diseminación de la información por todos los departamentos de la empresa y 3 la capacidad de respuesta de la organización ante esta información (capacidad para el diseño y la ejecución de los planes); proponen la escala MARKOR para medir estos tres componentes. La orientación al mercado tiene su base en el supuesto de que las firmas ganan y sostienen una ventaja competitiva si conocen las necesidades cambiantes del mercado; la mayor parte de los estudios ponen énfasis en la importancia de la adopción de este enfoque que se relaciona con el desempeño de las empresas y con la cultura emprendedora, el efecto sobre los empleados y los análisis comparativos de organizaciones que aplican o no la orientación al mercado (Montiel, 2018; Bucktowar et. al., 2015). 
El concepto de desempeño de las organizaciones está referido al proceso gestionable que integra una serie de componentes, tales como el talento, la estructura, el ambiente de negocios y los resultados esperados en la organización (Alsos y Ljunggren, 2017), otro concepto indica que el desempeño se refiere al crecimiento financiero de las empresas que toma en cuenta el cálculo del retorno sobre la inversión, activos y utilidades de periodo (Rust et. al., 2004); también se toma en cuenta el dinamismo del mercado, la innovación, la aplicación de estrategias de diferenciación y calculan el volumen de ventas para determinar el desempeño organizacional (McKelvie et. al., 2018; Bucktowar et. al., 2015); finalmente, estudios más cualitativos toman como indicador la percepción del directivo sobre el crecimiento de ventas, la rentabilidad de la empresa y su crecimiento integral (Ynzunza e Izar, 2013). El modelo de Khedhaouria et. al. (2015) da mayor importancia a la naturaleza cualitativa del análisis de rentabilidad debido a la dificultad de acceso a la información financiera de las empresas; la medición del desempeño se hace basándose en dos atributos: percepción del empresario del crecimiento de la empresa (rentabilidad) y percepción sobre el nivel de ventas.

En este estudio se ha tomado en cuenta el análisis del desempeño organizacional basado en tres dimensiones la económica, medición basada en la percepción del empresario en relación al crecimiento del nivel de ventas, ganancias y rentabilidad (Khedhaouria et. al., 2015); la dimensión ambiental está basada en la gestión del medio ambiente que debe realizar las empresas para lograr reducir sus costes y en el mediano plazo conseguir aumentar su valor financiero (Hobson y Essex, 2001) y una tercera dimensión referida a lo social enfocada hacia la inversión en actividades de responsabilidad social empresarial que provocan el aumento de valor de la empresa y la rentabilidad (Padilla et. al., 2017; Kang et. al., 2010). Con los antecedentes teóricos expuestos; se formulan las siguientes hipótesis: 1) la orientación al mercado (OM) se relaciona positivamente con el desempeño organizacional (DO); 2) el perfil femenino (PF) se relaciona positivamente con la orientación al mercado; y 3) el perfil femenino se relaciona positivamente con el desempeño organizacional.

\section{METODOLOGÍA}

Para cumplir con el objetivo de este artículo, se utilizó una metodología cuantitativa, mediante un diseño no experimental y de corte transversal; se presenta en una primera parte la descripción de la población estudiada y en la segunda se explica la validez del instrumento aplicado para la medición de las variables analizadas.

\section{Población sujeto de estudio}

Se consideró como población a todas las mujeres gerentes-propietarias en la industria hotelera de dos ciudades patrimoniales del sur de Bolivia. Tomando en cuenta que el $85 \%$ de los negocios hoteleros están a cargo de mujeres se realizó un censo a 190 gerentes propietarias (El restante 15\% son hoteles cuyo dueño y gerente no es mujer). Para tener acceso a la información se visitó cada uno de los negocios hoteleros incluidos en el estudio, se solicitó el consentimiento para administrar el cuestionario y se garantizó la confidencialidad y privacidad de los datos e información proporcionada, en síntesis se acudió a 112 hoteles en Sucre y a 78 en Potosí.

\section{Instrumento y escala de medición}

El instrumento de estudio fue construido tomando en cuenta los antecedentes teóricos de las variables analizadas y las relaciones entre sí, está estructurado en tres partes: La primera está referida a los datos generales de la empresa hotelera, tomando en cuenta información sobre cambios en los ingresos por los servicios, número de empleados, antigüedad del hotel, número de camas y promedio de camas ocupadas por mes. La segunda presenta los datos de la empresaria proporcionando información sobre su edad, años de experiencia general, años de experiencia específica, nivel de formación, estado civil, número de hijos, miembros de su familia, aporte compartido al ingreso familiar (Significa dependencia y ayuda económica). La tercera parte mide los distintos constructos del modelo, se usó una escala Likert de 5 puntos ( $1=$ "totalmente en desacuerdo" a $5=$ "totalmente en acuerdo" donde el valor 3 es interpretado como punto de neutral); en el instrumento se consideraron escalas ya validadas por otros autores tal como se presenta en la Tabla 1.

Se realizó el análisis de fiabilidad y validez con el apoyo de software SPSS, utilizando el método de Máxima Verosimilitud y rotación Varimax; los resultados del análisis de validez y confiabilidad del instrumento se presentan en la Tabla 2 en la cual se pueden observar que los valores del índice Kaiser-Meyer-Olkin (KMO) para las tres variables son mayores de 0,8 y las pruebas de esfericidad de Bartlett son significativas; esto representa una validez aceptable del instrumento; el análisis de confiabilidad por medio del Alfa de Cronbach arroja un resultado de 0,88 para PF, 0,859 para OM y 0,86 para DO; por tanto estos indicadores de fiabilidad y validez del constructo indican que existe una consistencia interna aceptable, superior al límite 
inferior permitido que oscila entre 0,60 y 0,70 ( Martínez y Fierro, 2018). Se aclara que para la validez de contenido, el cuestionario, fue presentado a profesionales expertos con la finalidad de recolectar sugerencias acerca de la redacción de los ítems; considerando las recomendaciones respectivas se procedió a realizar cambios en algunos ítems respecto a la forma de expresión y además a aumentar algunos reactivos que no se habían tomado en cuenta.

Tabla 1. Escala de medición

\begin{tabular}{|c|l|l|c|l|}
\hline Variable & \multicolumn{1}{|c|}{ Factores } & \multicolumn{1}{|c|}{ Items } & Noítems & \multicolumn{1}{|c|}{ Fuente bibliográfica } \\
\hline \multirow{2}{*}{ PF } & Capital humano y estilos de & PF1, PF2, PF3, PF4, PF5, PF6, & \multirow{2}{*}{10} & Millán et. al.(2014) \\
Gómez et. al.,(2015) \\
\cline { 3 - 5 } & aprendizaje & PF7, PF8, PF9, PF10 & 2 & Liñan y Santos (2006) \\
\hline \multirow{3}{*}{ OM } & Capital social & PF11, PF12 & 4 & Narver y Slater (1990) \\
& Orientación al cliente & OM1, OM2, OM3, OM4, OM5, & 3 & Kohli y Jaworski (1990) \\
& Corientación a la competencia & OM6, OM7, OM8, OM9, OM10 & 3 & \\
& Coordinación interfuncional & & 4 & Khedhaouria et. al.(2015) \\
\cline { 3 - 5 } DO & Dimensión económica & DO1, DO2, DO3, DO4 & 4 & Hobson y Essex (2001) \\
\cline { 2 - 5 } & Dimensión ambiental & DO5, DO6, DO7, DO8 & 4 & Kang (2010) \\
& Dimensión social & DO9, DO10, DO11,DO12 &
\end{tabular}

Tabla 2. Medida Kaiser-Meyer-Olkin de adecuación de muestreo y prueba de esfericidad de Bartlett

\begin{tabular}{|c|c|c|c|c|}
\hline \multicolumn{2}{|c|}{ Detalle } & PF & OM & DO \\
\hline \multicolumn{2}{|c|}{$\begin{array}{l}\text { Medida Kaiser-Meyer-Olkin de adecuación de } \\
\text { muestreo }\end{array}$} & ,831 & ,805 & 0,893 \\
\hline \multirow{3}{*}{$\begin{array}{c}\text { Prueba de esfericidad } \\
\text { de Bartlett }\end{array}$} & Aprox. Chi-cuadrado & 1519,768 & 1110,146 & 2386,460 \\
\hline & $\mathrm{Gl}$ & 66 & 45 & 66 \\
\hline & Sig. &, 000 &, 000 &, 000 \\
\hline \multicolumn{2}{|r|}{ Alfa de Cronbach } & 0.88 & 0.859 & 0.86 \\
\hline \multicolumn{2}{|r|}{ Rho A } & 0.889 & 0.863 & 0.859 \\
\hline \multicolumn{2}{|r|}{ Fiabilidad compuesta } & 0.887 & 0.861 & 0.858 \\
\hline \multicolumn{2}{|c|}{ Varianza extraída media (AVE) } & 0.613 & 0.608 & 0.548 \\
\hline
\end{tabular}

Por otro lado los resultados de la validez discriminante se presentan en la Tabla 3, señalan que las medidas son válidas pues las correlaciones entre los mismos constructos presentan los valores máximos en la matriz.

Tabla 3. Validez discriminante

\begin{tabular}{|l|l|l|l|}
\hline Variables & Desempeño organizacional & Orientación al mercado & Perfil femenino \\
\hline Desempeño organizacional & 0,740 & & \\
\hline Orientación al mercado & 0,659 & 0,779 & \\
\hline Perfil femenino & 0,554 & 0,690 & 0,783 \\
\hline
\end{tabular}

Posterior al análisis de fiabilidad y validez del instrumento se realizó el análisis confirmatorio y el contraste de hipótesis, en esta etapa se aplicó el análisis de ecuaciones estructurales a través del software SmarthPLS.

\section{RESULTADOS}

Los resultados de la aplicación del instrumento señalan que la edad promedio de las mujeres empresarias fue de 46,75 años, con una desviación estándar de 12,47 años y un rango de edad de 66 años que osciló entre los 23 y 89. La tenencia de hijos está en un promedio de 2,54 hijos y los miembros de familia que dependen de su ingreso son 2,76 en promedio. Los años de experiencia general son en promedio de 19,68 años y los años de experiencia específica en el rubro hotelero son de 12,81 años. Los datos de las empresas hoteleras incluidas en el estudio señalan que tienen un promedio de 6,03 empleados, tienen una antigüedad promedio de 13,03 años y disponen con un número de 35,74 camas. En la Tabla 4 se presentan las características demográficas de la población de estudio.

El modelo de medición fue probado utilizando el método matemático de mínimos cuadrados parciales (PLS) los resultados muestran la relación de las tres variables: la orientación al mercado (OM), el desempeño organizacional (DO) y el perfil femenino (PF) (Figura 1). 


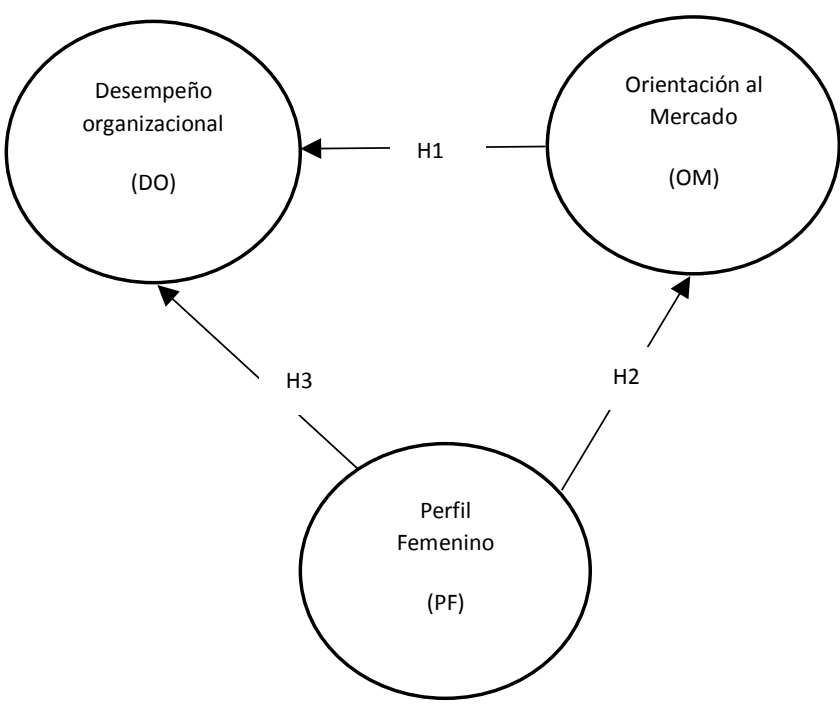

Fig. 1. Modelo propuesto e hipótesis

Tabla 4. Características de las mujeres participantes en el estudio

\begin{tabular}{|l|c|c|}
\hline Nivel educativo & Frecuencia & Porcentaje \\
\hline Primaria & 2 & 1,1 \\
\hline Secundaria & 44 & 23,2 \\
\hline Carrera Técnica o Comercial & 44 & 23,2 \\
\hline Licenciatura & 89 & 46,8 \\
\hline Posgrado & 11 & 5,8 \\
\hline Estado Civil & Frecuencia & Porcentaje \\
\hline Soltera & 24 & 12,6 \\
\hline Casada & 109 & 57,4 \\
\hline Conviviente & 24 & 12,6 \\
\hline Divorciada & 20 & 10,5 \\
\hline Viuda & 13 & 6,8 \\
\hline Aporte compartido al ingreso familiar & Frecuencia & Porcentaje \\
\hline Con nadie & 26 & 13,7 \\
\hline Pareja & 108 & 56,8 \\
\hline Hijos mayores & 33 & 17,4 \\
\hline Padres o hermanos & 10 & 5,3 \\
\hline Otros parientes & 13 & 6,8 \\
\hline
\end{tabular}

Con el fin de contrastar las hipótesis se tomó en cuenta los dos índices básicos: $\mathrm{R}^{2}$ (varianza explicada) (Tabla 6) y los coeficientes path estandarizados ( $\beta$ ) (Tabla 5). El primero indica que la varianza del constructo que es explicada por el modelo debería ser mayor o igual a 0.1 . El segundo índice, el coeficiente $\beta$ representa los coeficientes path o pesos de regresión estandarizados; para ser considerados significativos y aceptar la hipótesis, los coeficientes path estandarizados deberían alcanzar al menos un valor de 0.2, e idealmente situarse por encima de 0.3. (Martínez y Fierro, 2018)

Posteriormente se generó un bootstrapping con 5000 casos para generar error estándar y t estadístico con lo cual se evaluó la relación de significancia estadística de los coeficientes path de manera no paramétrica. Los resultados demuestran las hipótesis $\mathrm{H} 1$ y H2. Además, los resultados en la Tabla 6 corroboran que el modelo estructural tiene relevancia predictiva satisfactoria para la variable $D O(Q 2=0.054)$ y $O M(Q 2=$ 0.298). El contraste de hipótesis y las relaciones entre las tres variables se presentan en la Tabla 5 y en la Figura 2.

Tabla 5. Constatación de las hipótesis

\begin{tabular}{|l|l|l|l|l|l|}
\hline Relaciones & Relación & $\begin{array}{l}\text { Coeficiente } \\
\text { path }(\beta)\end{array}$ & $\begin{array}{l}\text { Valor t } \\
\text { (bootstrap) }\end{array}$ & $\begin{array}{l}\text { P } \\
\text { valores }\end{array}$ & $\begin{array}{l}\text { Soportada/ } \\
\text { No soportada }\end{array}$ \\
\hline Orientación al Mercado $\rightarrow$ Desempeño Organizacional & Positiva & 0.529 & 5.097 & 0,000 & Soportada \\
\hline Perfil femenino $\rightarrow$ Orientación al mercado & Positiva & 0.690 & 12.535 & 0,000 & Soportada \\
\hline Perfil femenino $\rightarrow$ Desempeño Organizacional & Positiva & 0.188 & 1.651 & 0,099 & No soportada \\
\hline
\end{tabular}


Por otro lado se calculó el $f^{2}$ que se utiliza para evaluar la contribución al $\mathrm{R}^{2}$, el efecto del $f^{2}$ en la relación entre PF y OM es alta mientras que entre PF y DO el efecto es bajo (Tabla 6 ).

Tabla 6. Efecto del $\mathrm{f}^{2}$

\begin{tabular}{|l|l|l|c|l|}
\hline Variables & $\mathrm{R}^{2}$ & $\mathrm{Q}^{2}$ & $\mathrm{f}^{2}$ & Percentile 95\% confidence interval \\
\hline DO & 0.453 & 0.054 & 0.034 & {$[0,420 ; 0,676] \mathrm{sig}$} \\
\hline OM & 0.476 & 0.298 & 0.910 & {$[0,572 ; 0,786] \mathrm{sig}$} \\
\hline
\end{tabular}

En cuanto a la bondad de ajuste del modelo, los resultados del $R^{2}$ muestran índices superiores a 0,1 ; si bien algunos autores indican que una varianza con valores cercanos a $50 \%$ es baja, otros estudios señalan que es representativa pues el rango considerado está entre $50 \%$ y $60 \%$; para Henson y Roberts (2006) esperar una varianza explicada mayor a ese rango es una expectativa poco realista. (Tabla 6).

Por otro lado los valores que comprueban el ajuste del modelo son: la prueba de Residuos Medios Estandarizados RME (Standardized Root Mean Square Residual, SRMSR) que mide la diferencia entre la matriz de correlación observada y la matriz de correlaciones implícita del modelo. Un buen ajuste del modelo no debe exceder de 0.08 , en este caso el ajuste se produce con valores de 0.067 . Otro valor medido es el Índice de Ajuste Normado IAN (Normed Fit Index, NFI); en este caso el valor alcanzado es de 0.789 que es bajo, pero se advierte que esto se genera cuando las muestras son pequeñas (Ullman, 2006) (Tabla 7).

Tabla 7 Indices de ajuste del modelo

\begin{tabular}{|l|r|r|}
\hline \multicolumn{1}{|c|}{ Indices } & Modelo saturado & Modelo estimado \\
\hline SRMR & 0.067 & 0.067 \\
\hline Chi-cuadrado & 360.338 & 360.338 \\
\hline NFI & 0.789 & 0.789 \\
\hline
\end{tabular}

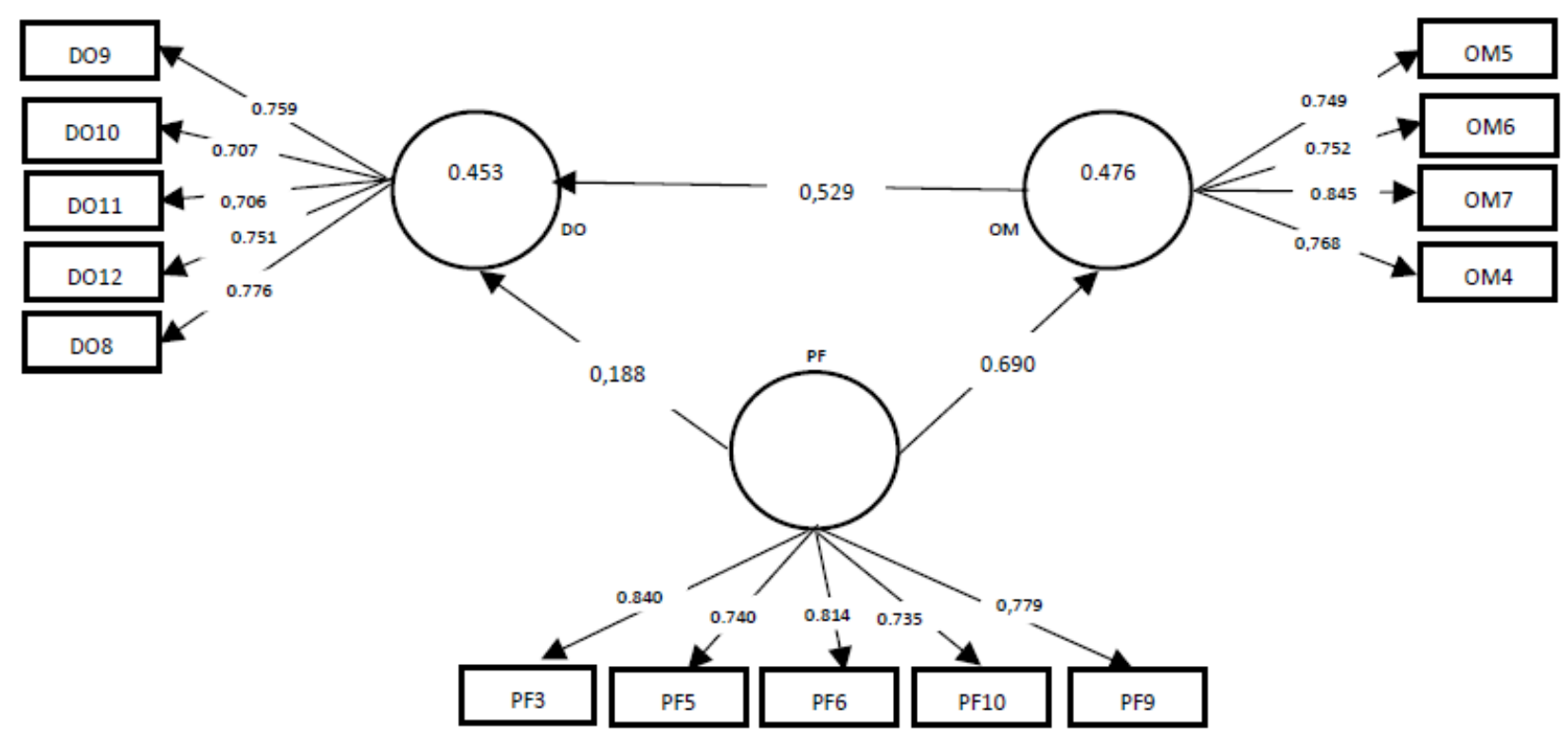

Fig. 2. Resultados del modelo aplicado

\section{DISCUSIÓN}

Existe una mayor incidencia entre el capital humano y la variable latente PF con relación al capital social; es decir que, en percepción de las mujeres gerentes propietarias, tiene una mayor incidencia su capital humano (el $76 \%$ tienen una carrera técnica y/o universitaria) en relación a su capital social (el $37 \%$ indica que ha tenido apoyo de su red social más cercana para aminorar la carga familiar y sólo el $5 \%$ perciben una red institucional que fomente su negocio); resultados que no coincide con el análisis de Alsos y Ljunggren (2017) cuando indica que las mujeres son las menos arriesgadas, las que mejor usan su capital social y las que menos forman su capital humano.

La orientación al mercado y el desempeño organizacional presentan una relación positiva y significativa, coincidiendo con los estudios de Ynzunza e Izar (2013) esta relación es significativa de acuerdo a la aplicación del modelo, lo cual permite deducir que la orientación al mercado impulsa y promueve la creación 
de un valor superior para los clientes y, como consecuencia de ello, se incide en la mejora del desempeño organizacional. Sobre la relación entre el perfil directivo de las mujeres y la orientación al mercado, la cual es significativa, se deduce que tiene que ver con la formación de su capital humano que incide en la orientación emprendedora y la proactividad para identificar necesidades de los clientes y oportunidades de negocios que han tenido las mujeres gerentes-propietarias en el sector hotelero. Al respecto y de acuerdo con lo que dicen Lomberg et. al., (2017) Y Berkhout et. al. (2016) el perfil directivo tiene que ver con un perfil de orientación emprendedora entendido como estilos y filosofía de gestión empresarial, con toma de riesgos y proactividad.

En lo que se refiere a la relación entre el perfil directivo femenino y el desempeño organizacional la relación no es significativa, esto permite interpretar que las mujeres no perciben el desempeño de su organización ya que en su mayoría están a cargo de empresas pequeñas y medianas de tipo familiar en un sector intensivo en mano de obra y orientado a los servicios. Sin embargo indirectamente por el cumplimiento de la hipótesis $\mathrm{H} 2$ se puede asumir que el perfil de las mujeres sí influye en el desempeño organizacional tomando en cuenta la variable orientación al mercado como mediadora, así lo expresa Montiel (2018) cuando indica que muchos estudios han llegado a la conclusión de que el carisma de los directivos, el estilo de liderazgo y la orientación emprendedora determinan la orientación al mercado y por tanto influyen en el desempeño organizacional.

\section{CONCLUSIONES}

1. En general se confirma una relación positiva entre las tres variables: perfil directivo femenino, orientación al mercado y desempeño organizacional percibida por las gerentes-propietarias de hoteles en las dos ciudades patrimoniales del sur de Bolivia.

2. Particularmente, existen dos implicaciones importantes en los hallazgos de este trabajo: primero los resultados revelan que el perfil directivo es un aspecto determinante para aplicar la orientación al mercado en las empresas y como consecuencia lograr un mejor desempeño organizacional, en este sentido es importante fortalecer los perfiles directivos de las gerentes propietarias de la industria hotelera en ciudades patrimoniales del sur de Bolivia. En segundo lugar los hacedores de políticas públicas para el sector hotelero deben brindar mayor atención al capital humano y social de las mujeres gerentes-propietarias, especialmente dirigiendo asistencia y capacitación a esta población y generando alianzas estratégicas que permitan mejorar la gestión de esta clase de organizaciones.

3. Sobre las limitaciones para esta investigación se debe indicar que se ha considerado sólo un país (Bolivia) y una industria (hoteles), esto no permite generalizar los resultados. Por otro lado el estudio toma en cuenta una muestra pequeña por las limitaciones del contexto en que se desarrolló la investigación (Ciudades de menos de 300.000 habitantes), finalmente otra limitación es que se sigue un enfoque de modelado que se centra más en la predicción que en la causalidad.

\section{REFERENCIAS}

Aldana-Bernal, J. C., y Bernal-Torres, C. A. El capital social y la integración de procesos en la gestión de las cadenas de abastecimiento en el sector real en Colombia. Información tecnológica, 30(5), 249-262 (2019).

Alsos, G. A., y Ljunggren, E. The role of gender in entrepreneur-investor relationships: A signaling theory approach. Entrepreneurship: Theory \& Practice, 41(4), 567-590 (2017).

Baù, M., Sieger, P., Eddleston, K. A. y Chirico, F. Fail but Try Again? The effects of age, gender, and multiple-owner experience on failed entrepreneurs' reentry. http://dx.doi:10.1111/etap.12233. Entrepreneurship: Theory \& Practice, 41(6), 909-941 (2017).

Behrens, J., y Patzelt, H. Corporate entrepreneurship managers' project terminations: Integrating portfolio-level, individual-level, and firm-level Effects. Entrepreneurship: Theory \& Practice, 40(4), 815-842 (2016).

Berkhout, P., Hartog, J., y Praag, M. Entrepreneurship and financial incentives of return, risk, and skew. Entrepreneurship: Theory \& Practice, 40(2), 249-268 (2016).

Bourdieu, P. Le capital social: notes provisoires. In le capital social. La Découverte, 29-34 (2006).

Bucktowar, R., Kocak, A., y Padachi, K. Entrepreneurial orientation, market orientation and networking: Impact on innovation and firm performance. Journal Of Developmental Entrepreneurship, 20(4) (2015).

De la Mora, A. E., Alarcón, G., y López, J. F. Capital social y disponibilidad de mano de obra calificada como impulsores de la competitividad de las empresas que forman parte de clústeres aeroespaciales. El caso de México https://dx.doi.org/10.4067/S0718-07642020000100171. Información Tecnológica, 31(1) (2020).

Gómez, R. J., Villarreal, S. M. y Villarreal, S. F. Las incapacidades de aprendizaje organizacional y su relación con los estilos de aprendizaje organizacional en la Mipyme. Contaduría y administración, 60(3), 686-702 (2015). 
Henson, R. K., y Roberts, J. K. Use of exploratory factor analysis in published research: Common errors and some comment on improved practice. http://dx.doi.org/10.1177/0013164405282485. Educational and Psychological measurement, 66(3), 393-416 (2006).

Hobson, K. y Essex, S. Sustainable tourism: A view from accommodation businesses. Service Industries Journal, 21(4), 133-146 (2011).

Huggins, R., Prokop, D., y Thompson, P. Entrepreneurship and the determinants of firm survival within regions: human capital, growth motivation and locational conditions. Entrepreneurship \& Regional Development, 29:3-4, $357-389$ (2017).

Kang, K., Lee, S., y Huh, C. Impacts of positive and negative corporate social responsibility activities on company performance in the hospitality industry. International Journal of Hospitality Management 29, 72-82 (2010).

Khedhaouria, A., Gurău, C., y Torrès, O. Creativity, self-efficacy, and small-firm performance: the mediating role of entrepreneurial orientation. http://dx.doi.org/10.1007/s11187-014-9608-y, 485-504. Small Business Economics, 44(3) (2015).

Kohli, A. K., y Jaworski, B. J. Market orientation: the construct, research propositions, and managerial implications. The Journal of Marketing, 1-18 (1990).

Liñán, F., y Santos, F. La influencia del capital social sobre los empresarios potenciales. Estudios de Economía Aplicada, Vol. 24, 459-489 (2006).

Lomberg, C., Urbig, D., Stöckmann, C., Marino, L. D. y Dickson, P. H. Entrepreneurial Orientation: The Dimensions' Shared Effects in Explaining Firm Performance. http://dx.doi:10.1111/etap.12237. Entrepreneurship: Theory \& Practice, 41(6), 973-998 (2017).

Lu, J. y Herremans, I. Board genderdiversity and environmental performance: An industries perspective. http://dx.doi.org/10.1002/. Bus Strat Env.1-16., 1-16 (2019).

Martínez, Á. M., y Fierro, M. E. Aplicación de la técnica PLS-SEM en la gestión del conocimiento: un enfoque técnico práctico. http://dx.doi: 10.23913/ride.v8i16.336. Revista Iberoamericana para la Investigación y el Desarrollo Educativo, 8(16), 130-164 (2018).

McKelvie, A., Wiklund, J., y Brattström, A. Externally acquired or internally generated? Knowledge development and perceived environmental dynamism in new venture innovation, http://dx.doi.org/10.1177/1042258717747056. Entrepreneurship Theory and Practice, 42(1), 24-46 (2018).

Millán, J. M., Congregado, E., y Román, C. Entrepreneurship persistence with and without personnel: The role of human capital and previous unemployment. International Entrepreneurship and Management Journal, 10(1), 187-206 (2014).

Montiel, C. H., Entrepreneurial orientation and market orientation: Systematic literature review and future research. http://dx.doi.org/10.1108/JRME-09-2017-0040. Journal of Research in Marketing and Entrepreneurship (2018).

Morales, C. C., Perfil de la mujer en la alta dirección en Colombia. Bogotá. Universidad Del Rosario (2011).

Naldi, L., Baù, M., Ahl, H., y Markowska, M. Gender (in) equality within the household and business start-up among mothers. http://dx.doi.org/10.1007/s11187-019-00275-1. Small Business Economics, 1-16 (2019).

Narver, J. C., y Slater, S. F. The effect of a market orientation on business profitability. The Journal of marketing, 20-35 (1990).

Padilla C. P., Arévalo D. X., Bustamante M. A. y Vidal C. L. Responsabilidad social empresarial y desempeño financiero en la industria del plástico en Ecuador. http://dx doi: dx.doi.org/10.4067/S. Información Tecnológica, 28(4), 93-102 (2017).

Querejazu, V., Zavaleta, D., y Mendizabal, J. Global emtrepreneurship monitor: Reporte Nacional Bolivia. Bolivia (2014).

Rust, R. T., Lemon, K. N. y Zeithaml, V. A. Return on marketing: Using customer equity to focus marketing strategy. journals.ama.org/doi/pdf/10.1509/jmkg.68.1.109.24030. Journal of marketing, 68(1), 109-127 (2004).

Santamaria, D., y Filis, G. Tourism demand and economic growth in Spain: New insights based on the yield curve. Tourism Management, 75, 447-459 (2019).

Ullman, J. B., Structural equation modeling: Reviewing the basics and moving forward. Journal of personality assessment, 87(1), 35-50 (2006).

Ventura, F. R., y Quero, G. M. Factores explicativos de la intención de emprender en la mujer. Aspectos diferenciales en la población universitaria según la variable género. Cuadernos de gestión, 127-149 (2012).

Ynzunza, C. C., y Izar, L. J., Estrategia, orientación al mercado y desempeño organizacional. Conciencia Tecnológica, 5-11 (2013). 